\title{
Correction to: Long-Short Temporal-Spatial Clues Excited Network for Robust Person Re-identification
}

\author{
Shuai $\mathrm{Li}^{1,2} \cdot$ Wenfeng Song $^{1}$ (1) $\cdot$ Zheng Fang $^{1} \cdot$ Jiaying Shi ${ }^{1} \cdot$ Aimin $\mathrm{Hao}^{1,2} \cdot$ Qinping Zhao $^{1,2} \cdot$ Hong Qin $^{3}$ \\ Published online: 6 July 2021 \\ ○) Springer Science+Business Media, LLC, part of Springer Nature 2021

\section{Correction to:} \\ International Journal of Computer Vision \\ Publisher's Note Springer Nature remains neutral with regard to \\ jurisdictional claims in published maps and institutional affiliations.
}

(2020) 128:2936-2961

https://doi.org/10.1007/s11263-020-01349-4

The original version of this article unfortunately contained a typographical error. The affiliation of co-author's Shuai Li's and Hong Qin was incorrect.

The second affiliation of Shuai Li is Peng Cheng Laboratory, Shenzhen, China, while Hong Qin should be with the Department of Computer Science, Stony Brook University (State University of New York), Stony Brook, New York 11794-2424, USA.

The original article can be found online at https://doi.org/10.1007/ s11263-020-01349-4.

Wenfeng Song songwenfenga@163.com

$\bowtie$ Hong Qin

qin@cs.stonybrook.edu

Shuai Li

lishuai@buaa.edu.cn

1 State Key Laboratory of Virtual Reality Technology and Systems, Beihang University, Beijing, China

2 Peng Cheng Laboratory, Shenzhen 518055, China

3 Department of Computer Science, Stony Brook University (State University of New York), Stony Brook, NY 11794-2424, USA 